\title{
Planning for Mixed Discrete Continuous Domains
}

\author{
Maria Fox \\ Department of Computer Science and Information Systems \\ University of Strathclyde, U.K.
}

\begin{abstract}
Mixed discrete-continuous systems are hybrid systems that exhibit both discrete changes of state, describable in terms of their logical and metric properties, and continuous numeric change describable in terms of differential equations. Continuous change occurs within a state as a consequence of one or more continuous processes being active in that state, whilst discrete change results in state transitions. Such hybrid systems are well-understood in the formal verification and real-time control communities.

Many real planning problems involve interaction with continuously changing values that directly affect both the validity and efficiency of plans. The problem of planning with continuous effects is harder than planning under the assumption of discrete change. The planner must be capable of reasoning about the evolution of continuous processes and their interactions with discrete state changes. For this reason, the standard approach to handling complex continuous effects in planning is to abstract them out of the domain model by lifting the representation to a level where all change can be seen as discrete.

In this talk we discuss progress we have made towards planning in mixed discrete-continuous domains. We begin by arguing that there are problems of critical interest to potential users of planning technology that cannot be adequately modelled under the assumption of discreteness. We then discuss an approach to planning in these domains that relies on the integration of a discrete planner with a continuous non-linear constraint solver. We present some results taken from a range of planning domains featuring continuous change. We discuss the future of this branch of planning and relate our work to the AI and OR literature.
\end{abstract}

